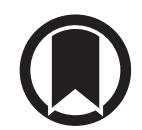

CrossMark

\title{
Wedge resection and radiofrequency ablation for stage I nonsmall cell lung cancer
}

\author{
Marcello C. Ambrogi ${ }^{1}$, Olivia Fanucchi ${ }^{2}$, Paolo Dini ${ }^{2}$, Franca Melfi ${ }^{2}$, \\ Federico Davini ${ }^{2}$, Marco Lucchi ${ }^{2}$, Gabriele Massimetti $^{3}$ and Alfredo Mussi ${ }^{1}$ \\ Affiliations: \\ ${ }^{1}$ Division of Thoracic Surgery, Department of Surgical, Medical, Molecular, and Critical Area Pathology, \\ University of Pisa, Pisa, Italy. \\ ${ }^{2}$ Division of Thoracic Surgery, Cardiac Thoracic and Vascular Department, Cisanello University Hospital, Pisa, Italy. \\ ${ }^{3}$ Division of Psychiatry, Department of Clinical and Experimental Medicine, University of Pisa, Pisa, Italy.
}

\section{Correspondence:}

Olivia Fanucchi, Cardiac Thoracic and Vascular Department, via Paradisa 2, 56124 Pisa, Italy.

E-mail: olivia.fanucchidafor.unipi.it

ABSTRACT The main aim of this study was to compare radiofrequency ablation (RFA) and wedge resection in terms of disease recurrence and survival, as intent-to-treat therapy for stage I nonsmall cell lung cancer (NSCLC) in marginal or non-surgical candidates.

121 high-risk patients, treated for stage I NSCLC with wedge resection $(n=59)$ or RFA $(n=62)$ in a 7-year period, were analysed. Age, sex, comorbidity score, performance status, forced expiratory volume in $1 \mathrm{~s}$ and forced vital capacity values, body mass index, T-stage and histology were evaluated as possible risk factors affecting disease recurrence and survival.

At a median follow-up of 36 and 42 months for wedge resection and for RFA ( $p=0.232$ ), local recurrence rate was 2 and 23\%, respectively ( $\mathrm{p}=0.002$ ). The 1-, 2- and 5-year overall survival (disease-free interval) rates were $100 \%$ (96\%), 96\% (90\%) and 52\% (76\%) for wedge resection, and 93\% (87\%), $72 \%$ $(63 \%)$, and $35 \%(55 \%)$ for RFA ( $p=0.044$ and $p=0.01$, respectively). None of the analysed parameters was found to be risk factor for disease recurrence and survival, except stage T2, which significantly affected disease-recurrence, overall and cancer-related survival and disease-free interval in the RFA group.

Whenever possible, surgical resection, even if limited, should be preferred due to its higher disease control and survival rates. RFA can be considered a valid option for inoperable patients, especially for those with stage T1N0.

@ERSpublications

Limited resection provides better oncologic outcomes in respect to RFA, but, for stage IA NSCLC, they seem equivalent http://ow.ly/Hn23G 


\section{Introduction}

The cornerstone of therapy of early-stage nonsmall cell lung cancer (NSCLC) is surgical resection with lobectomy and systematic lymph node evaluation [1]. However, in an ageing population, poor lung function and comorbidities could preclude surgery or create a significant risk of complications after surgery. Sub-lobar resections were offered with a curative intent to marginal surgical candidates, due to their preservation of lung function [2-4]. However, many patients with early-stage NSCLC are unable to undergo any operation because of comorbidities [5]. Given these data, some alternative non-surgical treatments have been developed during previous years. One such therapy is percutaneous radiofrequency ablation (RFA), which is defined as a procedure with negligible mortality, low morbidity, short hospital stay and gain in quality of life [6,7] providing acceptable results in terms of survival in non-surgical candidates [8].

In this scenario, we decided to compare RFA and wedge resection as intent-to-treat therapy in non-surgical or marginal-surgical patients: the primary end-point was to compare survivals and disease control, and the secondary end-point was to evaluate possible risk factors affecting disease-recurrence and survival.

\section{Material and methods}

Our Institutional Ethic Committee approved the study (approval number 3616). 121 patients were treated at our Department for their stage I NSCLC (T1/T2N0M0) with RFA $(n=62)$ or wedge resection $(n=59)$, during the period 2006-2012. Patients who underwent RFA belong mainly to a previous prospective study [8]. Patients who underwent wedge resection were retrospectively selected from our surgical database (Ormaweb; Dedalus S.p.A., Florence, Italy), including exclusively those with pathological stage I disease.

Demographic and clinical data of each patient was recorded. The Adult Co-Morbidity Evaluation scoring system (ACE-27) was used to stratify population based on co-morbidities. Adverse events were recorded using the Common Terminology Criteria for Adverse Events, version 4.03 [9]. Pre-operative and post-operative quality of life (evaluated 6 months after the treatment) was assessed according to Karnofsky scale performance status.

\section{Wedge resection}

All patients were evaluated unfit for lobectomy. The decision to perform a wedge resection was taken by an expert thoracic surgeon together with an anaesthesiologist, a pneumologist and an oncologist, based on medical conditions and/or pulmonary reserve (table 1).

All procedures were completed with a curative intent. Surgical approach (muscle-sparing thoracotomy or thoracoscopy) was chosen based on lesion size and depth: 30 patients underwent thoracoscopy. Of the remaining 29 patients who underwent thoracotomy, two were scheduled for thoracoscopic resection, but underwent thoracotomy due to nodule localisation failure (one case) and presence of tenacious pleural adhesions (one case).

\section{Radiofrequency ablation}

All patients had a biopsy-proven NSCLC. All patients were evaluated to be unable to tolerate any surgical intervention, based on cardiopulmonary reserve and/or medical co-morbidities (table 1). Additional selection criteria are reported in a previous paper [8]. The majority of the lesions (59 out of 62) were peripheral, according to the Radiation Therapy Oncology Group criteria [10], the remaining three lesions were central, but always at a minimum distance of $1 \mathrm{~cm}$ from large vessels and the bronchus, as per inclusion criteria [8].

All procedures were performed with a curative intent, under conscious sedation and local anaesthesia, utilising a radiofrequency generator (RITA-Model 1500/1500X; AngioDynamics, Latham, NY, USA). 10 patients were treated up to four times for the same lesion due to persistence or recurrence of the disease, for a total of 79 RFAs. The mean (range) interval between ablations was 3 (1-12) months. The procedures were performed using computed tomography (CT) or ultrasonography guidance (five cases), according to a protocol based on tumour size, as reported in a previous paper [8].

\section{Follow-up}

For wedge resection patients, a chest radiograph was performed 3 months after surgery, and subsequently chest and upper abdomen CT scan was performed every 6 months. For RFA patients, radiological follow-up consisted of a contrast-enhanced CT at 1,3, and 6 months after the procedure, and then with an interval of every 6 months. The assessment of target tumour response was based on CT analysis of lesion size, lesion geometry and lesion enhancement. Considering that the objective of the RFA protocol was to encompass the tumour with an ablation zone of at least $1 \mathrm{~cm}$ thickness, the 1-month follow-up CT scan (in which the high-density area representing the ablation zone was usually larger than the native tumour) 


\begin{tabular}{|c|c|c|c|}
\hline & Wedge resection & RFA & Correlation \\
\hline Age years & $70(56-83)$ & $76(60-88)$ & $t=-3.60 ; p=0.041^{\#}$ \\
\hline Sex M/F n & $46 / 13$ & $45 / 17$ & Chi-squared $=0.23 ; p=0.635^{\natural}$ \\
\hline Baseline performance status $^{+}$ & $90(80-100)$ & $80(60-90)$ & $t=7.69 ; p=0.017^{\#}$ \\
\hline \multicolumn{4}{|l|}{ Comorbidities $\mathrm{n}^{\S}$} \\
\hline Cerebrovascular disease & 3 & 5 & \\
\hline Congestive heart failure & 6 & 11 & \\
\hline Coronary artery disease ${ }^{f}$ & 15 & 19 & \\
\hline Chronic obstructive pulmonary disease & 37 & 32 & \\
\hline Dementia & 2 & 3 & \\
\hline Diabetes mellitus & 9 & 15 & \\
\hline Hepatic disease & 7 & 8 & \\
\hline Hypertension & 17 & 11 & \\
\hline Peripheral arterial disease & 3 & 9 & \\
\hline Renal disease & 6 & 11 & \\
\hline Ulcer disease & 4 & 3 & \\
\hline Other & 5 & 4 & \\
\hline \multicolumn{4}{|l|}{ Adult Comorbidity Evaluation 27 score } \\
\hline \multicolumn{4}{|l|}{0} \\
\hline 1 & 28 & & \\
\hline 2 & 16 & 20 & \\
\hline 3 & 15 & 42 & \\
\hline $1-2$ versus 3 & 42 versus 15 & 20 versus 42 & Chi-squared $=20.4 ; p<0.001^{\Uparrow}$ \\
\hline FEV $1 \%$ predicted & $47(40-56)$ & $49.5(27-111)$ & $t=-2.55 ; p<0.001^{\#}$ \\
\hline FVC $\%$ predicted & $57(39-56)$ & $59(35-101)$ & $t=-2.36 ; p<0.001^{\#}$ \\
\hline $\mathrm{BMI} \mathrm{kg} \cdot \mathrm{m}^{-2}$ & $24(20-30)$ & $26(20-30)$ & $t=-1.51 ; p=0.242^{\#}$ \\
\hline TNM stage $n^{\# \#}$ & & & Chi-squared $=1.38 ; p=0.241^{\natural}$ \\
\hline T1NOMO & 16 & 23 & \\
\hline T2NOMO & 43 & 39 & \\
\hline Tumour size mm & $26(12-33)$ & $23(12-43)$ & $t=-1.362 ; p=0.175^{\#}$ \\
\hline Histology $n$ & & & Chi-squared $=0.05 ; p=0.825^{\natural}$ \\
\hline Adenocarcinoma & 35 & 38 & \\
\hline Squamous cell carcinoma & 24 & 24 & \\
\hline \multicolumn{4}{|c|}{$\begin{array}{l}\text { Data are presented as median (range), unless otherwise stated. }{ }^{\#}: t \text { test; }{ }^{~}{ }^{*} \text { : Chi-squared or Fisher's exact } \\
\text { test where appropriate; }{ }^{+} \text {: according to Karnofsky scale; }{ }^{\S} \text { : one patient can be affected by one or more } \\
\text { comorbidities; }{ }^{f} \text { : coronary artery disease was defined as a positive exercise testing or prior myocardial } \\
\text { infarction or prior coronary bypass. \#\#: for wedge resection, } 11 \text { out of } 59 \text { T2 cases were due to visceral } \\
\text { pleural invasion, all patients underwent lymph node sampling (median number of station } 3 \text { (range } 2-5 \text { ), } \\
\text { median number of lymph node } 9 \text { (range } 6-14 \text { )), and for RFA, } N \text { stage was clinical. }\end{array}$} \\
\hline
\end{tabular}

was taken as a term of reference. Nodules showing at least a $30 \%$ decrease in longest diameter compared with the diameter measured at the 1-month CT, no evidence of tumour growth from the zone of ablation, and no evidence of contrast enhancement were assumed to have undergone complete ablation. The threshold of $30 \%$ is consistent with the Response Evaluation Criteria in Solid Tumours (RECIST) and was aimed at preventing overestimation of response due to measurement variability [11]. Conversely, nodules showing with at least a $20 \%$ increase in longest diameter in any of the follow-up CT studies (as per RECIST, taking as reference the smallest diameter measured at any time point), evidence of tumour growth from the zone of ablation or intra-tumoural contrast uptake (equal/greater than preoperative but greater than 25 Hounsfield Units) were assumed to have disease recurrence.

During the follow-up, a positron emission tomography (PET) scan was performed when the aforementioned criteria were not clearly satisfied, and never in the early post-treatment period, due to the presence of inflammatory tissue surrounding the thermal injury $[12,13]$, such as suggested by the study of Yoo et al. [14]. Additionally, a PET scan was used to assess the occurrence of distant (extra-cranial) metastasis.

In case of multiple RFAs, the follow-up analysis for survival and disease-free interval was calculated from the latest treatment. Local recurrence (LR) was defined as evidence of tumour recurrence near the site of surgical resection (for patients undergoing wedge resection), near or in the site of thermal ablation (for patients undergoing RFA), and within the same lobe (for both groups). Distant recurrence (DR) was defined as evidence of tumour disease in any other thoracic or extra-thoracic site. 


\section{Statistical analysis}

Statistical analysis was performed using the software STATISTICA 7.0 for Windows (Stat-Soft Inc., Tulsa, OK, USA). Differences and similarities between the two cohorts were assessed using unpaired test and the Chi-squared test, for continuous data and categorical measures, respectively.

LR, DR, freedom from any recurrence (FFR), overall survival, cancer-related survival (CRS), and disease-free interval (DFI) were analysed using the Kaplan-Meier method, and possible differences between the two groups were assessed with log-rank test. We also investigated, both by univariate and multivariate analysis, possible correlations between T-stage, visceral pleural invasion (only for wedge resection), type of surgical approach (open thoracotomy versus thoracoscopy, only for wedge resection), histology (adenocarcinoma versus squamous cell carcinoma) and LR and DR in both groups. In addition to these factors, age, sex, pre-operative performance status, percentage of predicted forced expiratory volume in $1 \mathrm{~s}(\mathrm{FEV} 1)$, percentage of predicted forced vital capacity (FVC), body mass index (BMI) and ACE-27 score were analysed for association with survival in both groups.

For univariate analysis, categorical variables were analysed using log-rank and continuous variables were analysed using Cox regression. Multivariate analysis was performed using Cox regression. The odds ratio (OR) and corresponding 95\% confidence intervals (CI) were reported for covariates. A p-value $<0.05$ was considered statistically-significant.

\section{Results}

In this retrospective study, RFA patients were older than surgical ones, with higher comorbidity score and worst performance status. These evidences are the consequence of the most important inclusion criteria for RFA: surgical contraindication. Lung function tests were better for RFA with respect to wedge resection. It may seem a contradiction, but the range values of pulmonary function can be clarifying. In the RFA group, the FEV1 values ranged between 27 and $111 \%$ of predicted: some of RFA patients had extremely poor lung function, but most of them had good lung function and were contraindicated to surgery due to non-pulmonary comorbidities, as suggested by the high ACE score.

There were no significant differences in T-stage, in tumour dimension and histology (table 1).

Table 2 summarises the post-treatment results, showing shorter hospitalisation and lower complication profile for RFA.

There were no statistically-significant difference between pre-operative and post-operative performance status in both groups ( $\mathrm{p}=0.564$ for wedge resection, $\mathrm{p}=0.672$ for RFA).

TABLE 2 Post-treatment outcomes of wedge resection and radiofrequency ablation (RFA)

\begin{tabular}{|c|c|c|c|}
\hline & Wedge resection & RFA & Correlation \\
\hline 30-day mortality \% & 0 & $0^{+}$ & \\
\hline 90-day mortality \% & 0 & $0^{+}$ & \\
\hline Morbidity & $16(27)$ & $13(16)^{+}$ & $\begin{array}{c}\text { Chi-squared=2.31; } \\
p=0.128^{\S}\end{array}$ \\
\hline Grade 1 adverse events" & $6(10)$ & $10(13)^{+}$ & $\begin{array}{c}\text { Chi-squared }=0.2 ; \\
p=0.651^{\S}\end{array}$ \\
\hline Details & $\begin{array}{l}6 \text { prolonged air leaking } \\
\qquad(>5 \text { days })\end{array}$ & $\begin{array}{c}4 \text { pleural effusion; } 5 \\
\text { pneumothorax; } 1 \text { chest } \\
\text { wall haematoma }\end{array}$ & \\
\hline Grade 2 adverse events" & $10(17)$ & $3(4)^{+}$ & $\mathrm{p}=0.01^{\S}$ \\
\hline Details & $\begin{array}{c}5 \text { atrial fibrillation, } 2 \text { wound } \\
\text { dehiscence, } 2 \text { anaemia, } \\
1 \text { urinary retention }\end{array}$ & $\begin{array}{l}3 \text { pneumothorax } \\
\text { requiring chest tube }\end{array}$ & \\
\hline $\begin{array}{l}\text { Post-treatment } \\
\text { hospital stay days }\end{array}$ & $6(4-22)$ & $2(1-4)^{+}$ & $\mathrm{t}=8.79 ; \mathrm{p}<0.001^{f}$ \\
\hline $\begin{array}{l}\text { Post-treatment } \\
\text { performance status }\end{array}$ & $90(80-100)$ & $80(60-90)$ & $t=7.34 ; p=0.021^{f}$ \\
\hline
\end{tabular}

Data are presented as $\mathrm{n}(\%)$ or median (range), unless otherwise stated. ${ }^{\text {* }}$ : Common Terminology Criteria for Adverse Event version 4.03; ๆ: according to Karnofky scale, assessed at our consulting room 6 months after the last treatment; ${ }^{+}$: calculated on the 79 RFA procedures; ${ }^{\S}$ : Fisher's exact test or Chi-squared test, where appropriate; ${ }^{f}$ : t-test. 


\section{Survival and risk factor analysis}

No patients were lost at follow-up. At a median follow-up time of 36 and 42 months for wedge resection and for RFA ( $\mathrm{p}=0.232)$, respectively, a statistically-significant difference was observed in LR ( $2 \%$ for wedge resection and $23 \%$ for RFA; $\mathrm{p}=0.002$ ) and in FFR ( $86 \%$ for wedge resection and $71 \%$ in $\mathrm{RFA} ; \mathrm{p}=0.010$ ). No significant difference was identified in DR between the two groups ( $12 \%$ in wedge resection and $11 \%$ in RFA; $p=0.621$ ). The 1-, 2- and 5-year overall survival (CRS) were 100\% (100\%), 96\% (98\%) and 52\% $(68 \%)$ for wedge resection, and $93 \%(100 \%), 72 \%(73 \%)$ and $35 \%$ (59\%) for RFA, with significant difference between the two groups ( $\mathrm{p}=0.044$ and $\mathrm{p}=0.024$; respectively) (figs 1 and 2). DFI at 1,2 and 5 years was 96,90 and $76 \%$ for wedge resection and 87,63 and $55 \%$ for RFA, with a statistically significant difference ( $\mathrm{p}=0.01)$ (fig. 3).

As a result of insufficient events in the wedge resection group, factors predictive of LR were not tested. No association was observed between histology, T-stage, visceral pleural invasion, type of surgical approach and DR or FFR for patients who underwent wedge resection. For patients who underwent RFA, histology was not correlated with LR, DR and FFR, while stage T1 was associated with lower LR $(\mathrm{p}<0.001)$, lower DR $(\mathrm{p}<0.001)$ and higher FFR $(\mathrm{p}<0.001)$, compared with stage T2.

With regard to the analysis of possible risk factors affecting survival of wedge resection patients, in both univariate and multivariate analysis, no association was observed between age, sex, performance status, percentage of predicted FEV1 and FVC values, BMI, ACE-27 score, histology, T-stage, visceral pleura invasion or type of surgical approach (table 3 ).

With regard to RFA, univariate and multivariate analyses showed no correlation between survival and age, sex, performance status, percentage of predicted FEV1 and FVC values, BMI, ACE-27 score or histology. Stage T1 was strongly related with better overall survival $(p=0.014)$, CRS $(p<0.001)$ and DFI $(p<0.001)$, by univariate analysis. Also multivariate analysis confirmed that T-stage significantly affected survival ( $p=0.042$; OR 5.13 95\% CI 1.06-24.83) (table 3). Given these data, we compared only stage IA patients, observing no statistically-significant difference between the two groups in terms of overall survival $(\mathrm{p}=0.499)$, CRS $(\mathrm{p}=0.386)$ and DFI $(\mathrm{p}=0.531): 1-, 2$-, and 5-year overall survival was 100,97 and $62 \%$ for wedge resection and 95,81 , and $52 \%$ for RFA.

\section{Discussion}

New approaches are currently being developed to treat early-stage NSCLC in an increasing population of high-risk and inoperable patients. Recently, CRABtree et al. [15] compared the results of three multi-institutional clinical trials (RTOG 0236 for stereotactic body radiation therapy (SBRT), ACOSOG Z4032 for sublobar resection with or without brachytherapy, ACOSOG Z4033 for RFA), for treatment of stage I NSCLC in high-risk patients. However, in the aforementioned paper, survival and recurrence data of RFA (51 patients) had not yet matured; thus, for RFA, the comparative analysis regarded only mortality ( $2 \%$ at 90 days), showing no statistical difference with the other treatments. Nevertheless, that paper showed a higher complication rate for sublobar resection compared with SBRT [15]. As expected, also in our study, wedge resection was associated with a higher complication profile compared with nonsurgical therapy, but both wedge resection and RFA appeared to be safe, with low morbidity (27 and 16\%, respectively), consistent with previous data $[3,4,6-8,16]$. Additionally, no significant changes between baseline and post-treatment quality of life were observed in either group. These facts could be considered the result of accurate patient evaluation, performed case by case, in a multidisciplinary setting.

FIGURE 1 Cumulative proportional overall survival for wedge resection and radiofrequency ablation.

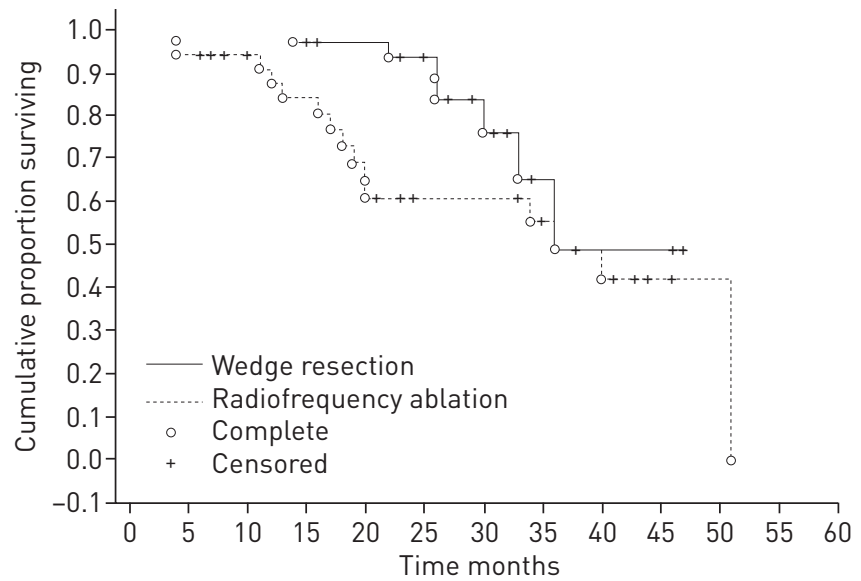




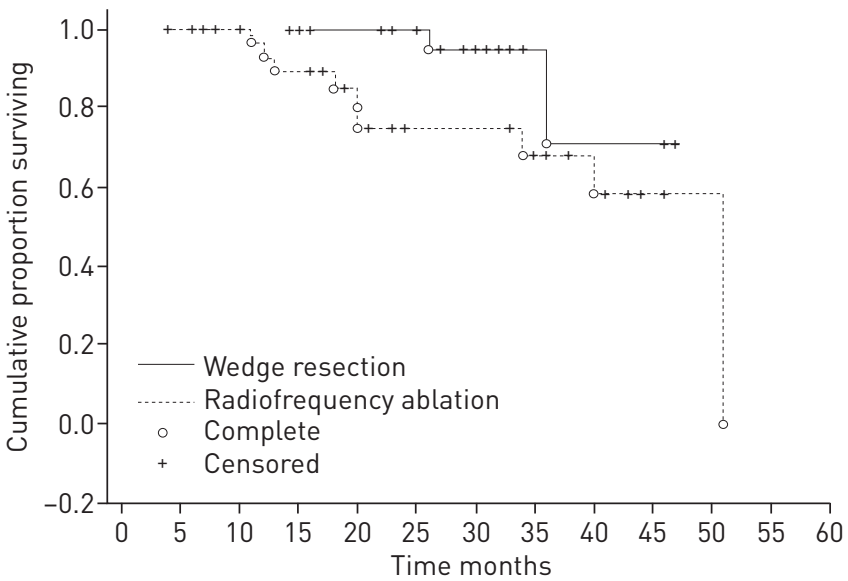

FIGURE 2 Cumulative proportional cancer-related survival for wedge resection and radiofrequency ablation.

However, comparing morbidity between different modalities of therapy is difficult, especially between surgical and nonsurgical treatments. For this reason, the comparison between RFA and SBRT is probably more appropriate. In the RFA patients, the most frequent complication was pneumothorax, such as reported in the present paper, ranging from 4.5 to $61 \%$, and generally it did not require a chest tube [ 7,8 , 16-19]. With regard to SBRT, the most frequent adverse event was pneumonitis, ranging from 9.7 to $66.1 \%$ [20-22], depending on the radiation dose, which generally varied from 48 to $60 \mathrm{~Gy}$, but ONISHI et al. [22] applied a dose up to $180 \mathrm{~Gy}$. Lung toxicities, related to the damage of normal lung tissue, can occur as acute or late event, and can make the patients oxygen dependent $(27.7 \%$ of patients at a median of 55.6 months after treatment, in the series of FAKIRIs et al. [23]). In the RFA series, no significant decline in pulmonary function was observed [8]. This fact can be partially explained by two facts. First, the application of RFA is independent of respiratory motion, in contrast to SBRT, even if different strategies were developed to reduce it (i.e. abdominal compression) [21, 22]; and secondly, radiofrequency energy spreads less in normal lung tissue, which has a higher level of impedance related to the presence of air, in respect to a solid lesion $[12,13]$. For this reason, RFA can be repeated for treatment of the same nodule, such as reported in this and other series $[8,16]$.

With regard to disease control, in our study, RFA was associated with higher LR in respect to wedge resection, while DR was similar between the two populations. Overall survival, CRS and DFI were significantly better for the surgical group, suggesting superior outcomes for resection, even if it is limited, as in wedge resection.

Concerning the risk-factors analysis, none of the investigated parameters influenced LR, DR and survival in either group, with the exception of stage T1 for RFA, which significantly affected LR and FFR, improving survival. This evidence suggests that RFA is more efficacious for small lesions, as supported previous studies $[7,8,16]$ and by the present analysis of stage T1N0 cases: no statistically significant difference was found between patients treated with RFA or wedge resection in terms of overall survival, CRS and DFI. These data were also confirmed by preliminary results recently published in an abstract of

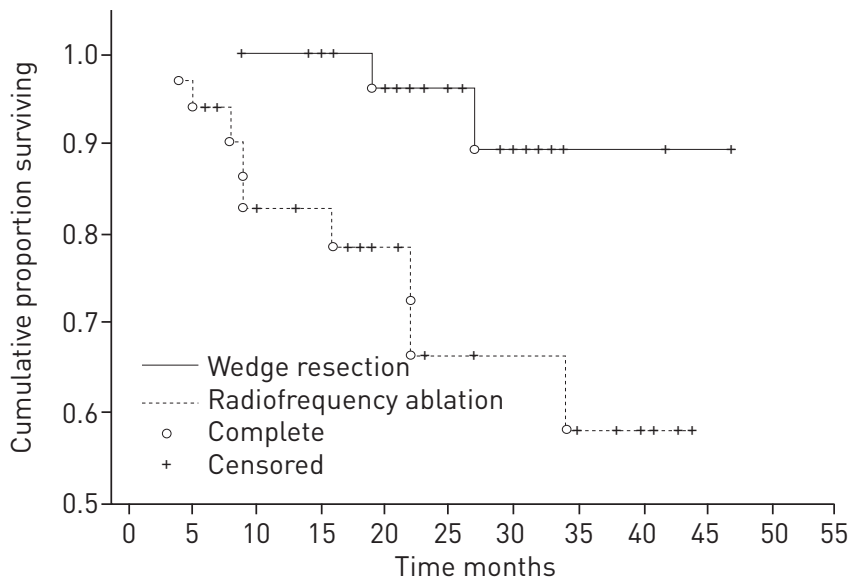

FIGURE 3 Cumulative proportional disease-free interval for wedge resection wedge resection and radiofrequency ablation. 
TABLE 3 Multivariate analysis (Cox regression) of possible risk factors affecting survival in wedge resection and radiofrequency ablation (RFA) patients

\begin{tabular}{|c|c|c|c|c|c|c|}
\hline \multirow[t]{2}{*}{ Risk factor } & \multicolumn{3}{|c|}{ Wedge resection } & \multicolumn{3}{|c|}{ RFA } \\
\hline & p-value & OR & $95 \% \mathrm{Cl}$ & p-value & OR & $95 \% \mathrm{Cl}$ \\
\hline Age & 0.150 & 1.076 & $0.974-1.188$ & 0.316 & 1.03 & $0.967-1.108$ \\
\hline Sex & 0.902 & 1.005 & $0.923-1.096$ & 0.321 & 0.486 & $0.197-1.162$ \\
\hline Performance status & 0.811 & 1.018 & $0.880-1.177$ & 0.224 & 0.965 & $0.912-1.022$ \\
\hline FEV1 \% predicted & 0.890 & 1.010 & $0.882-1.155$ & 0.095 & 1.025 & $0.675-1.044$ \\
\hline FVC $\%$ predicted & 0.783 & 1.221 & $0.652-1.332$ & 0.632 & 1.432 & $0.756-1.551$ \\
\hline BMI & 0.549 & 1.065 & $0.867-1.307$ & 0.869 & 0.989 & $0.866-1.129$ \\
\hline ACE-27 & 0.702 & 1.268 & $0.376-4.283$ & 0.334 & 1.558 & $0.634-3.825$ \\
\hline Histology & 0.712 & 0.787 & $0.220-2.816$ & 0.103 & 0.479 & $0.198-1.161$ \\
\hline T-stage & 0.577 & 1.532 & $0.342-6.852$ & 0.042 & 5.138 & $1.063-24.839$ \\
\hline Surgical approach & 0.856 & 1.002 & $0.947-1.326$ & NA & NA & NA \\
\hline Visceral pleural invasion & 0.744 & 1.121 & $0.874-1.206$ & NA & NA & NA \\
\hline
\end{tabular}

OR: odds ratio; $95 \% \mathrm{Cl}$ : 95\% confidential interval; $\mathrm{FEV} 1$ : forced expiratory volume in $1 \mathrm{~s}$; FVC: forced vital capacity; BMI: body mass index; ACE-27: Adult Co-Morbidity Evaluation scoring system; NA: not applicable.

the ACOSOG Z4033 study. In that abstract, performance status was associated with increased survival, but this was not found in our analysis [24].

To our knowledge, our study is the largest that directly compares survival of RFA and wedge resection, as intent-to-treat local therapies for stage I NSCLC. In 2012, LeE et al. [25] compared RFA with surgery, obtaining no statistically-significant difference in overall survival (table 4).

However, this study regarded small series, whose stage ranged from I to IV, furthermore the type of surgical resection, anatomic or non-anatomic, was not specified [25]. KIM et al. [26] compared RFA ( $n=8)$ with lobectomy and pneumonectomy $(\mathrm{n}=14)$ after a matching process based on age, sex, tumour-nodemetastasis stage and time to treatment. They obtained a 5 -year survival rate of $25 \%$ for RFA and $67 \%$ for surgery, with no statistically significant difference. The only study comparing sublobar resection $(\mathrm{n}=25)$ with RFA $(\mathrm{n}=12)$ for treatment of stage I NSCLC, was the ZEMLYAK et al. [27] study. They noted a trend towards higher recurrence in RFA and longer cancer-free interval for sublobar resection, although there was no statistically-significant difference, probably due to the small sample size. These results, as well as ours, are comparable with the results of different studies that separately analysed the outcomes of limited resection and RFA (table 4).

Nevertheless, the results of SBRT must also be considered. FAKIRIs et al. [23] in a prospective study on 70 stage I NSCLC patients, obtained a $90 \%$ overall survival at 1 year and $43 \%$ at 3 years, but this rate decreased to $20 \%$ at 5 years. Better results were reported by RICARDI et al. [20] who obtained 1-, 2- and 3 -year overall survival rates of 94, 81.6 and 68\%, respectively, on 196 patients. The study of RICARDI et al. also showed better overall and cancer-related survival for stage IA, both by univariate and multivariate analysis. These data are similar to those obtained with RFA, such as suggested by the present paper and other studies $[8,24]$. In a large cohort study on 245 stage I NSCLC patients, ONISH et al. [22] reported a 5 -year overall survival rates of $47 \%$, but this percentage increased to $90 \%$ when the authors considered only stage IA, medically operable patients, treated with an high-dose irradiation (>100 Gr) (87 patients). In a recent retrospective study, GRILLs et al. [21] compared SBRT and wedge resection for treatment of stage I NSCLC, obtaining significant improved overall survival for the wedge resection group, but no difference in terms of cancer-related survival.

The present study suggests that wedge resection could provide better oncologic outcomes in respect to RFA, leading, as a consequence, to prefer surgical resection when possible. SBRT seems to give good results; however, the approach for SBRT is not uniform across centres: the optimal radiation dose and number of fractions required are still not clearly defined.

Additionally, another key-point must be emphasised: lymph node sampling can be performed at the time of surgical intervention, allowing identification of unsuspected nodal disease and a more precise staging. The RFA option, in spite its higher disease recurrence, should be considered a valid option for inoperable patients, due to its low invasiveness, repeatability, low complications profile and short hospital stay. This is particularly true in stage T1N0 disease, for which RFA appears to be equivalent to wedge resection in terms of disease-free interval and survival. 
TABLE 4 Results of surgical resection, radiofrequency ablation (RFA) and stereotactic body radiation therapy (SBRT) for the treatment of stage I nonsmall cell lung cancer

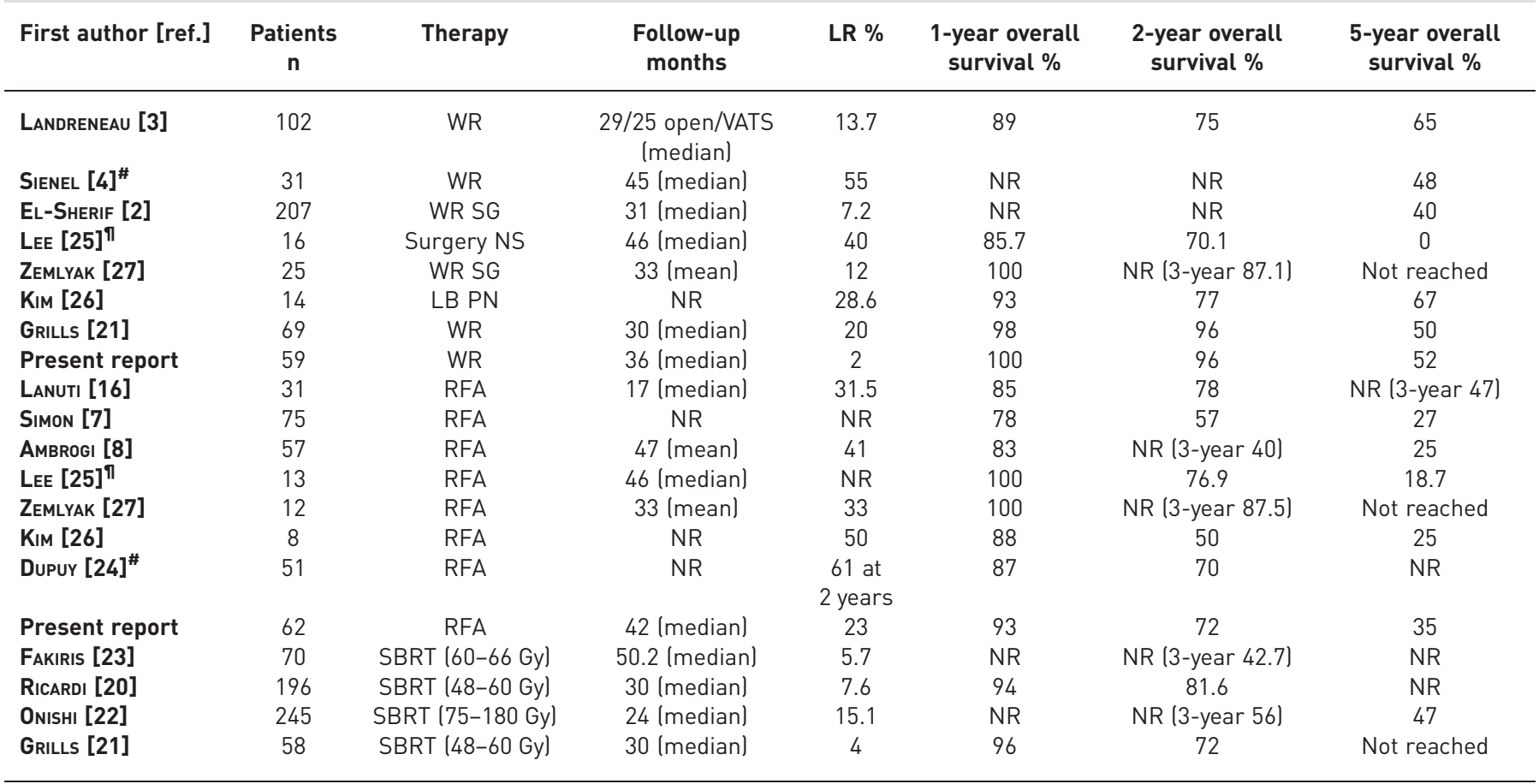

LR: local recurrence; WR: wedge resection; VATS: video-assisted thoracic surgery; NR: not reported; SG: segmentectomy; NS: not specified; LB: lobectomy; PN: pneumonectomy; SBRT: stereotactic body radiation therapy. " : Only stage IA; ๆ : stage I and II.

Despite our interesting results, we acknowledge some limits to this research. Even if RFA patients were treated in a prospective trial, the direct comparison with wedge resection was performed retrospectively. As a result of the retrospective nature of the study and of the different inclusion criteria for the two groups, patients undergoing RFA were older, with lower performance status and higher comorbidity score in respect to wedge resection, as expected by the fact that they were medically inoperable. This result underlines the necessity of specifically designed trials not only to compare different therapies, but also to stratify those patients considered at high risk. However, in this study, the two populations were well balanced in terms of histology and tumour dimension. Also disease stage was similar between the two groups, although the RFA patients were unavoidably clinically staged. Another critical point is related to the follow-up evaluation of local recurrence, which can be more difficult to detect in the RFA group due to the presence of scar tissue. For this reason, the assessment of target tumour strictly followed precise criteria, as reported above. Finally, there is the lack of a direct comparison with a cohort of patients treated with SBRT. Unluckily, this relatively-new promising technique is not available at our hospital, thus the comparison was performed using data from the literature.

In conclusion, the results of this study, with all limits of a retrospective non-randomised trial, confirm that surgical resection, even if limited to a wedge resection, has a better outcome compared with RFA and should therefore be preferred whenever possible, excluding only patients that are truly inoperable. Nevertheless, the debate seems open for patients with stage IA disease. In these cases, in fact, RFA seems to have equivalent outcomes compared with wedge resection, thus the selection of patients is more challenging due to the acceptable risk level, which depends also on the different success rate of the non-surgical alternative therapies. Further prospective randomised studies are necessary, in order to clearly compare the outcomes of different modality therapies, but also to better define patients considered at high risk.

\section{References}

1 Ginsberg RJ, Rubenstein LV. Randomized trial of lobectomy versus limited resection for T1 N0 nonsmall cell lung cancer. Lung Cancer Study Group. Ann Thorac Surg 1995; 60: 615-623.

2 El-Sherif A, Gooding WE, Santos R, et al. Outcomes of sublobar resection versus lobectomy for stage I nonsmall cell lung cancer: a 13-year analysis. Ann Thorac Surg 2006; 82: 408-416.

3 Landreneau R, Sugarbaker DJ, Mack MJ, et al. Wedge resection versus lobectomy for stage I (T1 N0 M0) nonsmall cell lung cancer. J Thorac Cardiovasc Surg 1997; 113: 691-700. 
4 Sienel W, Dango S, Kirschbaum A, et al. Sublobar resection in stage IA nonsmall cell lung cancer: segmentectomies result in significantly better cancer-related survival than wedge resections. Eur J Cardiothorac Sur 2008; 33: 728-734

5 Mery CM, Pappas AN, Bueno R, et al. Similar long-term survival of elderly patients with nonsmall cell lung cancer treated with lobectomy or wedge resection within the surveillance, epidemiology, and end results database. Chest 2005; 128: 237-245.

6 Steinke K, Sewell PE, Dupuy DE, et al. Pulmonary radiofrequency ablation: an international study survey. Anticancer Res 2004; 24: 339-343.

7 Simon CJ, Dupuy DE, Di Petrillo TA, et al. Pulmonary radiofrequency ablation: long term safety and efficacy in 153 patients. Radiology 2007; 243: 268-275.

8 Ambrogi MC, Fanucchi O, Cioni R, et al. Long-term results of radiofrequency ablation treatment of stage I nonsmall cell lung cancer: a prospective intention-to-treat study. J Thorac Oncol 2011; 6: 2044-2051.

9 Common Terminology Criteria for Adverse Events version 4.03. US Department of Health and Human Services, National Institutes of Health, National Cancer Institute, 2010.

10 www.rtog.org/ClinicalTrials/ProtocolTable/StudyDetails.aspx?study=0236. Date last updated November $2,2011$. Date last accessed: February 1, 2015.

11 Eisenhauer EA, Therasse P, Bogaerts J, et al. New response evaluation criteria in solid tumours: revised RECIST guideline (version 1.1). Eur J Cancer 2009; 45: 228-247.

12 Ambrogi MC, Fontanini G, Cioni R, et al. Biologic effects of radiofrequency thermal ablation on nonsmall cell lung cancer: results of a pilot study. J Thorac Cardiovasc Surg 2006; 131: 1002-1006.

13 Stainke K, Habicht JM, Thomsen S, et al. CT-guided radiofrequency ablation of a pulmonary metastasis followed by surgical resection. Cardiovasc Intervent Radiol 2002; 25: 543-546.

14 Yoo DC, Dupuy DE, Hillman SL, et al. Radiofrequency ablation of medically inoperable stage IA nonsmall cell lung cancer: are early posttreatment PET findings predictive for treatment outcome? AJR Am J Roentgenol 2011; 197: 334-340.

15 Crabtree T, Puri V, Timmerman R, et al. Treatment of stage I lung cancer in high-risk and inoperable patients: Comparison of prospective clinical trials using stereotactic body radiotherapy (RTOG 0236), sublobar resection (ACOSOG Z4032), and radiofrequency ablation (ACOSOG Z4033). J Thorac Cardiovasc Surg 2013; 145: 692-699.

16 Lanuti M, Sharma A, Digumarthy SR, et al. Radiofrequency ablation for treatment of medically inoperable stage I nonsmall cell lung cancer. J Thorac Cardiovasc Surg 2009; 137: 160-166.

17 Suh RD, Wallace $\mathrm{AB}$, Sheehan RE, et al. Unresectable pulmonary malignancies: CT-guided percutaneous radiofrequency ablation--preliminary results. Radiology 2003; 229: 821-829.

18 Thanos L, Mylona S, Pomoni M, et al. Percutaneous radiofrequency thermal ablation of primary and metastatic lung tumors. Eur J Cardiothorac Surg 2006; 30: 797-800.

19 Hiraki T, Tajiri N, Mimura H, et al. Pneumothorax, pleural effusion, and chest tube placement after radiofrequency ablation of lung tumors: incidence and risk factors. Radiology 2006; 241: 275-283.

20 Ricardi U, Frezza G, Filippi AR, et al. Stereotactic Ablative Radiotherapy for stage I histologically proven nonsmall cell lung cancer: an Italian multicenter observational study. Lung Cancer 2014; 84: 248-253.

21 Grills IS, Mangona VS, Welsh R, et al. Outcomes after stereotactic lung radiotherapy or wedge resection for stage I non-small-cell lung cancer. J Clin Oncol 2010; 28: 928-935.

22 Onishi H Araki T Shirato H, et al. Stereotactic hypofractionated high-dose irradiation for stage I nonsmall cell lung carcinoma: Clinical outcomes in 245 subjects in a Japanese multiisnstitutional study. Cancer 2004; 101: 1623-1631

23 Fakiris AJ, McGarry RC, Yiannoutsos CT, et al. Stereotactic body radiation therapy for early-stage nonsmall-cell lung carcinoma: four-year results of a prospective phase II study. Int J Radiat Oncol Biol Phys 2009; 75: 677-682.

24 Dupuy D, Fernando H, Hillman S, et al. Radiofrequency Ablation of Stage 1A NSCLC in Medically Inoperable Patients: Results from ACOSOG Z4033 (Alliance), an NCI Funded Multicenter Trial. Chest 2013; 144: 86A-86A.

25 Lee H, Jin GY, Han YM, et al. Comparison of survival rate in primary nonsmall-cell lung cancer among elderly patients treated with radiofrequency ablation, surgery, or chemotherapy. Cardiovasc Intervent Radiol 2012; 35: $343-350$.

26 Kim SR, Han HJ, Park SJ, et al. Comparison between surgery and radiofrequency ablation for stage I nonsmall cell lung cancer. Eur J Radiol 2012; 8: 395-399.

27 Zemlyak A, Moore WH, Bilfinger TV. Comparison of survival after sublobar resections and ablative therapies for stage I nonsmall cell lung cancer. J Am Coll Surg 2010; 211: 68-72. 